\title{
Interseccionalidad y homosexualidad en Los delitos insignificantes y Contra natura, de Álvaro Pombo
}

Recibido: 24/1/2016

Aceptado: 19/9/2016

\section{RESUMEN:}

Las dos principales novelas de temática homosexual de Álvaro Pombo, Los delitos insignificantes (1986) y Contra natura (2005), coinciden en un cierto tipo de conceptualización de la homosexualidad que el propio autor llegó a calificar como "homosexualidad homófoba". En este artículo se discute el final trágico de ambas novelas (el suicidio del protagonista homosexual) a la luz del concepto de interseccionalidad, que describe la superposición y engarzamiento de discriminaciones múltiples, y que en el caso de Pombo no puede desligarse del contexto político de gradual normalización de las sexualidades no heterosexuales.

PALABRAS CLAVE: Álvaro Pombo, homosexualidad, homofobia, interseccionalidad, normalización de la homosexualidad, narrativa gay.

Intersectionality and homosexuality in two novels by Álvaro Pombo: Los delitos insignificantes and Contra Natura.

\footnotetext{
ABSTRACT:

Two of Álvaro Pombo's most influential gay novels, Los delitos insignificantes (1986) and Contra natura (2005), share a common understanding of homosexuality which Pombo himself referred to as "homophobic homosexuality". This paper considers the main gay character's suicide that comes at the conclusion of both novels. It makes use of the notion of interseccionality (overlay and interlocking of multiple systems
} 
of oppression), which in Pombo's case is closely related to Spain's political context of

gradual normalisation of non-heterosexual sexualities.

KEY WORDS: Keywords: Álvaro Pombo, homosexuality, homophobia, interseccio-

nality, homosexual normalisation, gay narrative.

Álvaro Pombo (Santander, 1939) es uno de los novelistas españoles cuya contribución a la creación y consolidación de la literatura española de temática homosexual ha sobrepasado ampliamente los límites impuestos por las modas y el mercado. Su cultivo de una narrativa indiscutible y plenamente homosexual se manifiesta en no pocas de sus novelas así como en sus colecciones de relatos en los que aparecen personajes y temáticas homosexuales, homosociales u homofílicos. Entre los títulos más significativos a este respecto se deberían mencionar los cuentos reunidos en Relatos sobre la falta de sustancia (1977) así como las novelas Los delitos insignificantes (1986), El metro de platino iridiado (1990), El cielo raso (2001) y Contra natura (2005).

Relatos sobre la falta de sustancia fue la obra con la que Álvaro Pombo se dio a conocer a su regreso a España en 1977, después de residir durante once años en Londres. El período de 1966 a 1977 fue, sin duda, de gran importancia para su formación literaria; en esos años escribió su primer poemario, Protocolos (1973), además de la mencionada colección de relatos. Fueron, también, años importantes para la historia de la cultura homosexual ya que en 1969 se produjeron en Nueva York los disturbios en torno al pub Stonewall Inn que habrían de encender la mecha del activismo LGBTQ. Las primeras manifestaciones del Orgullo Gay tuvieron lugar en Nueva York ese mismo año; en Londres, coincidiendo con la estancia de Pombo, tuvieron lugar en noviembre de 1970. Pronto se crearon varias organizaciones en defensa de los derechos de los homosexuales, entre las que destaca por su influencia internacional el Gay Liberation Front, que operó en Nueva York entre 1969 y 1972 y en Londres a partir de 1970. En España se creó el clandestino Movimiento Español de Liberación Homosexual en 1970, cuando la Ley de Peligrosidad y Rehabili- 
tación Social que condenaba explícitamente la homosexualidad aún estaba plenamente vigente. El MELH se disolvió en 1974. Al año siguiente surgió el Front d'Alliberament Gai de Catalunya, que en junio de 1977 organizó la primera manifestación reivindicativa de la que existe constancia en la historia del país.

Aunque años más tarde Pombo sostendría que su primera declaración pública de homosexualidad tuvo lugar en 1977 con la publicación de Relatos sobre la falta de sustancia ${ }^{1}$, lo cierto es que el escritor mantuvo una actitud pública extremadamente discreta durante muchos años. Su primera declaración inequívoca al respecto se puede datar en 2003, cuando en una entrevista publicada por la revista de temática LGBTQ Zero se manifestó abiertamente homosexual ${ }^{2}$. La figura pública de Pombo adoptó nuevos y destacados relieves a partir de esa fecha, no sólo por el hecho de alzarse con varios de los premios literarios más prestigiosos y con mayor proyección mediática del panorama literario español ${ }^{3}$, sino también por el inicio de una actividad política en

1 “Salí del armario sin ayuda de colectivo ninguno en 1977, con la publicación de mi primer libro de relatos (Relatos sobre la falta de sustancia)" (Pombo 2005b: 110). Sin embargo, tal publicación sólo podría considerarse como una salida del armario en un sentido implícito o tácito, ya que a pesar de que varios de los relatos incluyen personajes homosexuales en ningún momento el narrador (no digamos ya el autor) toma partido o declara su propia sexualidad (Martínez Expósito 2004: 118-128). En todo caso, le cabe a Pombo el mérito de ser el primer académico de la Española en salir públicamente del armario: “Fue no el primer gay en la Academia (obviamente, tan nutrida) pero sí en salir públicamente del armario como académico electo e investido. En suma, el primer respetable académico que posee en sus vitrinas el torso viril del premio Shangay" (Villena 2006).

\section{Revista Zero, 2003, número 50}

3 Entre otros, el Premio Herralde en 1983 por El héroe de las mansardas de Mansard (y finalista, ese mismo año, con El hijo adoptivo), el Premio de la Crítica en 1991 por El metro de platino iridiado, el Premio Nacional de Narrativa en 1997 por Donde las mujeres, el Premio Fastenrath en 1997 por La cuadratura del círculo, el Premio de la Fundación José Manuel Lara Hernández en 2001 por El cielo raso, el Premio Planeta en 2006 por La fortuna de Matilda Turpin, y el Premio Nadal en 2012 por El temblor del héroe. 
el seno del partido Unión Progreso y Democracia. A todo ello se une su trabajo en medios de comunicación de difusión nacional (como el programa Espejo Público de Antena 3). Lo más destacable, en lo que respecta al tema de la homosexualidad, es que en su vida pública Pombo ha adoptado de manera consistente una postura extremadamente crítica contra un cierto tipo de cultura homosexual militante con la que se identifican sectores del activismo LGBTQ. Esta postura le ha granjeado todo tipo de críticas desde esos sectores que son, precisamente, los que más decisivamente han contribuido a definir los contornos del debate público sobre los derechos de homosexuales y transexuales. En el centro de la controversia pombiana se encuentra el tema del matrimonio homosexual, que se ha erigido como asunto crucial del pensamiento homosexual en los comienzos del siglo XXI: un tema central que articula en torno a sí la gran mayoría de los debates públicos sobre todo aquello relativo a los discursos, los conceptos y las experiencias no heterosexuales (de manera similar a como en tiempos pretéritos lo hicieran la despatologización y la despenalización de los actos sexuales entre personas del mismo sexo y, más aún, la pandemia del sida). Estrechamente relacionadas con el matrimonio igualitario ${ }^{4} \mathrm{y}$ con los debates que en el año 2005 condujeron a su aprobación parlamentaria ${ }^{5}$, existen otras manifestaciones de esa misma cultura con la que Pombo se siente claramente a disgusto; entre ellas, muy notablemente, las celebraciones del Orgullo Gay, que en Madrid alcanzaron dimensiones desconocidas a partir de 2005, año en el que se alcan-

4 "Ahora empezamos a inventar para este amor [homosexual] nuevos relatos y nuevos conceptos. No puede ser, por lo tanto, reducido a la idea de amor matrimonial ni artificial. El concepto de matrimonio debe ser reservado para las uniones de heterosexuales. Hay que inventar conceptos nuevos. Y no se hace ningún servicio a la causa homosexual adaptando, poco más o menos, lo que ya hay a las grandes novedades que se avecinan" (Pombo 2005b: 113).

5 El PSOE presentó en junio de 2004 una proposición de ley con la que pretendía abrir la institución matrimonial a cónyuges del mismo sexo. El proyecto de ley se tramitó en abril de 2005 y finalmente la Ley 13/2005 fue aprobada el 2 de julio de ese mismo año. 
zaron por vez primera los dos millones de participantes, y que Pombo descalifica como una grosera simplificación que responde principalmente a motivaciones comerciales ${ }^{6}$.

La figura de Pombo representó, en sus comienzos literarios, la actitud del homosexual agobiado por sentimientos de culpa (Martínez Expósito 2004: 151-174). Durante los debates sobre el matrimonio igualitario adoptó posturas abiertamente críticas no sólo sobre el matrimonio sino sobre las profundas implicaciones que la aplicación de ese término y ese modelo tendrían para el mundo homosexual. Por estas razones, Pombo se ha convertido en uno de los más conspicuos oponentes a las agendas normalizadoras que la mayoría de los activistas LGBTQ han venido desarrollando en las últimas dos décadas. Para los defensores de la normalización, Pombo mantiene posturas contrarias a ese tipo de políticas porque en el fondo sigue sometido a su propia homofobia internalizada ${ }^{7}$. Es sin duda esta combinación de factores (su posicionamiento crítico sobre las políticas igualitarias y la reacción negativa del activismo ante su presunta endohomofobia) lo que ha llevado a Pombo a marcar distancias, practicando lo que él mismo denomina "homosexualidad homófoba" (Undurraga 2011) y declarándose como un "homosexual antiguo", "pre-gay", marcado por unas experiencias vitales que "reflejan la clase de homosexualidad que he vivido: era una imposibilidad. Uno no salía del armario nunca. Ni yo ni nadie. Hacías lo que podías" (Pombo en Rocamora 2009).

6 "Te convido a que vengas a Madrid a ver cómo es un día del orgullo gay: [...] es grotesco. Los osos van con las chupas con una raja en el culo ridículo. Que te tiren condones y vaselinas desde las carrozas es ridículo y además reduce la homosexualidad a una sola clase de homosexualidad: la gay [...] Pero no tienen sentido del humor, no se puede hacer una puta broma con la homosexualidad, ipor favor!" (Pombo en Undurraga 2011).

7 Véase, a modo de ejemplo, el ataque ad hominem del activista Enrique Hinojosa firmado con pseudónimo: Lily, Shangay, "La endohomofobia de Álvaro Pombo", Público, 15 de noviembre de 2011, <http://goo.gl/wG53Xh>. 
Gentes de mi generación nacidos alrededor del año 39 [...] fuimos también educados [...] en el existencialismo poético y filosófico. Una de las ideas de entonces fue la de autenticidad. Frente a la existencia inauténtica (el célebre decir lo que se dice, hacer lo que se hace, heideggeriano y sartreano), nosotros vivimos la ética de la responsabilidad personal, del compromiso [...] De aquí que viera mis propias inclinaciones homosexuales en estos términos y no en términos de entretenimiento o de búsqueda de pareja o parejas. No digo que esto fuera lo mejor o lo más inteligente o la única posibilidad: sólo digo que, en mi caso, autenticidad y realidad se presentaron siempre enfrentadas a irrealidad estética (gozo, felicidad) y superficialidad. Esto significa que yo viví (y creo que en esto coincido con la experiencia de toda mi generación) la homosexualidad como un difícil y enredoso asunto que, en virtud de mi sentido del compromiso y de la autenticidad, yo estaba obligado a hacer mío a toda costa. (Pombo 2005a: 557-558).

\section{De Los delitos insignificantes (1986) a Contra natura (2005)}

Los delitos insignificantes narra el romance de Ortega, escritor homosexual de mediana edad, y Quirós, atractivo joven en paro en el Madrid de los años ochenta. El tono optimista inicial se va ensombreciendo a medida que el elemento erótico cobra mayor importancia. Tras ser penetrado analmente por Quirós, algo que nunca antes había ocurrido, Ortega se suicida lanzándose por el balcón de su casa.

En Contra natura, el editor jubilado Javier Salazar entabla una relación con el joven Ramón Durán. La relación se complica con la aparición de un tercer personaje, Juanjo Garnacho, antiguo profesor de Ramón, que está casado con una mujer a la que oculta sus inquietudes homosexuales. Y se complementa con un cuarto personaje, Paco Allende, amigo de la misma edad de Javier pero con un sentido moral mucho más definido. Más que la línea narrativa de estos romances cruzados (que culminan con el suicidio de Javier Salazar), la novela explora las diferentes vivencias homosexuales de personajes nacidos durante el franquismo $\mathrm{y}$, frente a ellas, las de los nacidos en democracia. 
En enero de 2006, apenas dos meses después del lanzamiento editorial de la novela Contra natura, Álvaro Pombo y José Antonio Marina participaron en una actividad patrocinada por la Fundación Juan March cuyo formato consistía en la presentación, por parte del narrador, de una conferencia a modo de poética personal seguida de un análisis o comentario por parte del crítico. En su presentación, Pombo incidió en una de sus ideas centrales sobre la naturaleza de la literatura en tanto que modo de conocimiento, lo que él denomina "verdad narrativa", diferenciada de otros tipos de conocimiento científico, filosófico o histórico ${ }^{8}$. Por su parte, Marina exploró la doble alma literaria de Pombo:

Bergson decía que en el fondo de la obra de cada escritor hay una única intuición que intenta desarrollar a través de sus libros. En Pombo creo que hay dos, que permiten organizar su poesía y sus novelísticas en dos grandes ciclos: el ciclo negativo y el ciclo positivo. Uno es pesimista, desconfiado, y percibe la falta de sustancia de los seres. Culmina con Los delitos insignificantes. El otro es exaltado, brillante, y canta la belleza de las cosas o la santidad de las personas. Su obra emblemática podría ser Protocolos para la rehabilitación del firmamento. (Marina 2006: 10).

La doble intuición que Marina percibe en Pombo se puede rastrear sin dificultad en la práctica totalidad de su obra, tanto poética como narrativa, y resulta particularmente útil para un estudio de los personajes de sus obras mayores. En algunas de sus novelas más complejas, donde los personajes son sometidos a procesos de introspección narrativa infrecuentes en la literatura contemporánea, el rastreo del doble hálito, el pesimista y el exaltado, permite claramente adscribir cada personaje en cada momento concreto de su experiencia a uno de esos ciclos. No es el caso de Los delitos insignificantes, que Marina identifica sin

8 Pombo ha reflexionado sobre la naturaleza gnoseológica de la literatura en varios textos ensayísticos entre los que cabe destacar su discurso de ingreso en la Real Academia (Pombo 2004). 
ambages como el culmen de la pulsión negativa, ya que los dos personajes centrales están marcados por una pasión homosexual que los envuelve en una espiral de violencia y autodestrucción como si se tratara de una predestinación cuyo control está fuera de sus manos y que gobierna la dirección de sus actos. Pero en Contra natura la doble vía encuentra un punto de contraposición y comparación que no es inusual en Pombo (en El metro de platino iridiado, por ejemplo, se contraponen a menudo un personaje excelsamente positivo y un turbio personaje, homosexual, que desciende a lo largo de la novela a su propio infierno interior), y que aparece en esta novela con una sorprendente intención didáctica. La novela, de hecho, culmina con un "Epílogo" en el que el autor reflexiona del siguiente modo:

En esta novela, en definitiva, se plantean dos modos radicales de vivir la experiencia amorosa homoerótica masculina. Uno de ellos es válido: el otro, inválido. Dejo a la inteligente decisión del lector decidir cuál de los dos modelos es aceptable. (Pombo 2005a, 561).

Así, si en Los delitos insignificantes asistimos a la culminación inequívoca de la pulsión negativa, en Contra natura encontramos "dos modos radicales", uno claramente alineado con la pulsión negativa y el otro con la positiva. Por lo tanto, cabe preguntarse qué ha ocurrido entre una y otra para que la perspectiva unitaria y radicalmente negativa de la primera haya dejado paso a una perspectiva doble en la segunda9. La cuestión resulta aún más significativa si se considera, además, el notable paralelismo narrativo que une ambas novelas: por ejemplo, la formación de parejas homoeróticas constituidas por un hombre maduro de buena posición económica y un joven de escasos recursos eco-

9 “Pombo's early works represented characters suffering from internalized homophobia and guilt [...] From the 1990s on, however, his ethics and literary approach changed to a more positive representation and self-acceptance of homosexuality" (Pérez Sánchez 2014: 453). 
nómicos pero sobrados encantos físicos; la frecuente focalización narrativa en la perspectiva del hombre mayor; la presencia de algunos personajes femeninos cuya función narrativa parece limitarse a la de subrayar la indudable homosexualidad del hombre mayor y la relativamente maleable bisexualidad del joven; $\mathrm{y}$, sobre todo, el desenlace trágico consistente en el suicidio del hombre mayor.

Lo realmente llamativo del tratamiento pombiano de la experiencia homosexual en estas novelas no es tanto la insistencia en unos ciertos diseños narrativos, en unos personajes más o menos tipificados que, con variantes, van reapareciendo en sus relatos, o en unas ciertas soluciones narrativas a los lugares clásicos de la literatura homosexual tales como la dinámica del armario, las diferentes homofobias, o el romance entre hombres de muy distinto estrato social, como la resistencia a reducir la homosexualidad a una experiencia más o menos homologada, normalizada, o estandarizada. Los homosexuales de Pombo configuran galerías de personajes muy diversos que parecen estar unidos, en algunos casos, por el mero hecho, circunstancial a veces, de su homosexualidad. Esta insistencia en la diversidad y multiplicidad de la experiencia homosexual no es en absoluto casual sino que responde a la íntima convicción de Pombo, repetidamente expresada, de que la homosexualidad no puede en modo alguno verse limitada a una serie de tipos, experiencias o tópicos convenientemente preparados para su normalización cultural.

En el momento de su aparición, Los delitos insignificantes fue duramente criticada por su "visión culposa y extenuantemente melodramática, plagada de sinsabores y prejuicios interiorizados sobre la sexualidad y el amor entre hombres" (Aliaga 1997:78). Se trataba de una visión excesivamente negativa de la homosexualidad, que Juan Vicente Aliaga, en su estudio sobre la narrativa homosexual española, encuadra entre las obras de ficción que implícita o explícitamente ligan la homosexualidad a todo tipo de características distópicas. En esta novela la negatividad homosexual adquiere toda su intensidad en la composición del 
personaje de Gonzalo Ortega, un escritor frustrado de mediana edad que improvisadamente entra en una relación con el atractivo y canallesco joven César Quirós. Para Aliaga, las reflexiones del escritor sobre la homosexualidad, la propia y la ajena, revelan una auténtica tortura interior cifrada en la desesperación, la vergüenza y la soledad:

En un Madrid ambientado, grosso modo, entre finales de los setenta e inicios de la década siguiente, la caracterización de Ortega no puede resultar más antipática, incluso repulsiva: un personaje atormentado, débil y sexófobo que, tras ser penetrado por vez primera, en medio de un angustioso tira y afloja con Quirós, acaba suicidándose. La deshumanización que desprende la descripción del narrador produce grima y despersonaliza al homosexual, cuya condición cosificada resuelve el final. (Aliaga 1997:78).

El suicidio de Gonzalo Ortega reproduce un lugar común literario: el desenlace tanático que, simbólica o físicamente, aparece con frecuencia en la literatura homosexual. El desenlace trágico de Los delitos insignificantes está ocasionado, en opinión de Alberto Mira, por una defectuosa o inexistente definición de la homosexualidad, pues al no comprender lo que es la homosexualidad, al carecer de un sistema significante en el que enmarcar sus actos, el escritor maduro y el joven buscavidas no son más que dos seres perdidos en un universo agobiante: "homosexual o no, es difícil ser feliz y llevar una vida plena en el mundo de este escritor" (Mira 1999: 579).

Sin embargo, teniendo en cuenta el momento histórico en el que la novela aparece, cabe una interpretación alternativa según la cual la novela constituiría una denuncia del hecho de que, a pesar de la abolición de la Ley de Peligrosidad Social, a mediados de los años ochenta todavía se piense en la homosexualidad como un delito. En este sentido (si bien sólo en éste) la novela de Pombo coincidiría con La ley del deseo (1987), película de Pedro Almodóvar estrenada pocos meses después de la publicación de 
Los delitos insignificantes: ambas obras parecen querer llamar la atención sobre la criminalización de la homosexualidad, uno de esos delitos sin víctima o delitos predelictuales (Lamo de Espinosa 1989) entre los que se contaban el consumo de drogas, la homosexualidad o la prostitución, que en décadas anteriores habían sido objeto de leyes preventivas como la Ley de Vagos y Maleantes o la Ley de Peligrosidad Social. Los movimientos a favor de la total despenalización de la homosexualidad dieron lugar a muchos episodios de gran intensidad mediática en los años setenta; por su parte, Pombo pedía, a su regreso a España, tratar el tema de la homosexualidad "sin gesticulaciones melodramáticas" (García Sánchez 1979). En este contexto, una interpretación históricamente situada de Los delitos insignificantes debería tener en cuenta esta declarada aspiración de Pombo, si bien esa naturalidad debería entenderse como defensa del ejercicio de la libertad sexual de cada individuo, más que como aplicación de las pautas normalizadoras que por entonces comenzaban a exigir los colectivos LGBTQ.

Si Los delitos insignificantes se enmarca en la dinámica cultural de la transición española y de la despenalización de la homosexualidad, Contra natura surge en un momento histórico muy distinto, que coincide con el auge de una cultura LGTBQ que lucha por el Orgullo y por el matrimonio entre personas del mismo sexo. Pombo participó muy activamente en los debates sobre el matrimonio homosexual con posturas públicas que, en cierto modo, aparecen ilustradas y discutidas en Contra natura ${ }^{10}$. En el momento de su aparición, Contra natura llamó la atención de la crítica por su coherencia con la trayectoria novelística de Pombo, caracterizada por la narrativización de la conciencia y la conciencia narrativa; coherencia estilística y estética con su narrativa anterior que se hace especialmente visible en los casos de

10 Contra natura fue galardonada con los premios Salambó y Ciutat de Barcelona, este último por "l'audàcia en el tractament de les relacions sentimentals i dels seus plantejaments morals i narratius" (http://www.bcn.cat/cultura/premisciutatbc n/2005/\#literesp). 
Los delitos insignificantes, El metro de platino iridiado y El cielo raso. También la complejidad del tema homosexual llamó la atención de los principales reseñadores, que subrayaron la pluralidad de homosexualidades representadas por personajes que viven su sexualidad desde estilos de vida y edades diferentes. Contra natura pretende dar voz a una generación de homosexuales, la de los nacidos en la posguerra que, como el propio Pombo, vivieron su sexualidad sin el apoyo de la ideología normalizadora de la que las generaciones post-Stonewall han hecho bandera. Las homosexualidades plurales de Contra natura incluyen, como no podía ser de otro modo, la de aquellos que como Javier Salazar, el acomodado editor jubilado que protagoniza la novela, recuerdan tiempos muy diferentes.

Las prohibiciones humedecían el apetito, exaltaban los deseos, agudizaban los ingenios eróticos, jla calle brillaba con sus turbios amores prohibidos!, pero la prohibición, la nocturnidad, el secreto, el secreto de toda aquella incipiente sociedad rosa, lo que más tarde había de denominarse -estúpidamente- el morbo, ¿no era, en definitiva, parte de la maravillosidad, de la deseabilidad de la situación? Contra Franco nos la meneábamos mejor. (Pombo 2005a, p. 292).

Además del prototipo encarnado por Javier Salazar, que viene a ser el del intelectual ególatra y manipulador (claro heredero de la tradición del malditismo homosexual), en Contra Natura aparecen otros tres tipos muy identificables: Paco Allende, homosexual de la misma edad de Javier Salazar pero mucho más integrado y que no concibe su sexualidad como problema; Ramón Durán, joven veinteañero que se identifica con la generación gay joven; $y$ Juanjo Garnacho, homosexual casado de doble vida.

José María Pozuelo elogia la novela por no sumarse a la banalización de la literatura homosexual convertida en mercancía o moda y por ir a contracorriente de "la creciente conversión de lo homosexual en un tipo de consumo [...] para definir su diferencia, en el caso paradójico de una entidad, como si ser gay o lesbiana 
pudiera convertirse en una marca, del mismo modo que un barrio, que un día del orgullo o fenómenos parecidos de escaparatismo" (Pozuelo 2006). Pozuelo saluda la novela, "cruda, explícita, tremenda", porque se esfuerza en abordar la homosexualidad "de otro modo, contra la moral de la trivialización, pensando un problema [...] y con una introspección psicológica en sus personajes como he conocido pocas" (ibid.). Marcos Eymar va aún más lejos al sostener que "probablemente nunca antes, en toda la literatura española, se había realizado un retrato tan exhaustivo y sincero de las relaciones homosexuales [...] Pombo ha querido escribir también una historia de la homosexualidad en España" (Eymar, 2006: 42). Frente a una literatura homosexual comercializada hasta la trivialización que Pozuelo identifica con escritores mediáticos como Boris Izaguirre y Jaime Bayly, se alza en su opinión la literatura de calidad de una estirpe de escritores cuya nómina desgrana el propio Pombo en el "Epílogo" de la novela:

Lo que es evidentemente incompatible con esto (la normalización burguesa) es una idea de la homosexualidad inspirada en Gide, Wilde, Proust, Verlaine y Rimbaud, Luis Cernuda, Whitman, García Lorca, E. M. Forster o Gore Vidal, por no hablar de Tennesse Williams o Truman Capote o Auden o Chistopher Isherwood. La lista interminable de homosexualidades no caseras se extendía hacia atrás hasta Teognis de Mégara y Sócrates y Platón y, hacia adelante, a toda la variopinta serie de homosexuales de nuestros días (Pombo 2005a: 418).

En un acto de presentación de la novela, Pombo declaró: “Me dan miedo las normalizaciones. Es bueno que haya igualdad y se luche contra la homofobia, pero tratar la homosexualidad como algo fácil en lo que todo sale bien no me parece correcto, porque no hay ninguna relación humana fácil" (Pombo en Morán 2005). La cuestión de la normalización homosexual afecta también, en la perspectiva de Pombo, a la jerarquía católica; y esta alusión directa a la Iglesia, que para Pombo es un asunto central y de gran importancia personal (Pérez Sánchez 2014: 453), apenas se 
encuentra siquiera mencionado en la mayoría de reseñas de la novela. El propio Pombo se encargó de recordar la cuestión en su entrevista a Winston Manrique:

La novela está escrita con una idea de contribuir a la discusión sobre este asunto de la homosexualidad. En ese sentido tiene una voluntad de actualidad. Contra natura es una toma de posición respecto a las posiciones oficiales de la Iglesia católica española y a la Iglesia en general. La condena eclesiástica de la homosexualidad es frívola, al igual que la condena del Papa a los seminaristas homosexuales. (Pombo en Manrique 2005).

Luis Prádanos, al señalar la existencia de una serie de referencias filosóficas y poéticas que parecen gobernar la dinámica de la ficción, menciona dos ideas que Pombo toma de Ortega: la realidad como perspectiva y la búsqueda de la verdad a través de la acumulación de perspectivas. De ahí, señala Prádanos, el interés de Pombo en crear una narración focalizada en una pluralidad de personajes y un narrador que a pesar de su aparente omnisciencia va dejando en el aire preguntas sin contestar. Prádanos lee, acertadamente, Contra natura en clave fenomenológica como un texto en el que la construcción ontológica del sujeto se revela imposible ya que el autoconocimiento implica su propia aniquilación. Prádanos parece establecer una relación implícita entre Contra natura y algunas ficciones pombianas de tema religioso (La cuadratura del círculo, Vida de San Francisco de Asís) cuando señala que la anulación kantiana del sujeto en lo relativo a la moral "empalma con la tradición mística española donde el erotismo se imbuye de religiosidad y la existencia auténtica sólo se consigue con la aniquilación del ser" (Prádanos, 2006: 909).

\section{Interseccionalidad}

La deliberada pluralidad con la que Pombo aborda la representación de la homosexualidad en Contra natura resulta particularmente apta para un enfoque interseccional. La idea de inter- 
seccionalidad se ha venido aplicando de manera casi exclusiva desde la teoría feminista para estudiar casos de mujeres que sufren múltiples discriminaciones: a la discriminación por motivos de género se pueden añadir, por ejemplo, discriminaciones por motivos de raza, lengua, sexualidad, clase social, discapacidad, condición de inmigrante, y otras. Su utilidad, sin embargo, sobrepasa con creces los límites del feminismo y se deja sentir en una amplia gama de estudios que de un modo u otro se sienten concernidos por las desigualdades estructurales que afectan a tipos muy variados de personas. La idea de interseccionalidad pone de manifiesto las relaciones recíprocas que mantienen entre sí diferentes sistemas generadores de desigualdad. La intersección original en este tipo de enfoque surgió de la consideración por parte de Kimberlé Crenshaw y Patricia Collins (1990) de que resultaba notablemente improcedente y en todo caso insuficiente analizar por separado las opresiones que las afroamericanas sufrían por su condición de género y por su condición racial. Lo que realmente ocurría, según Collins, era que ambas opresiones estaban relacionadas entre sí, funcionaban de una manera en cierto modo orgánica, y no se podían separar analíticamente sin perder en el proceso de observación elementos centrales del sistema de opresiones múltiples que se trataba de definir. Naturalmente, una vez que el sistema de opresiones múltiples se define de manera más precisa salta a la vista que no se trata sólo del género y la raza, sino también la sexualidad, la clase socioeconómica, y toda una pluralidad de criterios que generan un entramado de opresiones. La perspectiva interseccional tiene el mérito de poner de relieve que tales opresiones no operan de manera autónoma sino que lo hacen orgánica y sistemáticamente.

El enfoque interseccional de los sistemas de opresión y desigualdad ha venido proporcionando una serie de instrumentos críticos de gran utilidad. Entre ellos deberíamos mencionar las nociones de simultaneidad, encrucijada y discriminación múltiple (Platero Méndez 2014: 60-65). La simultaneidad, como concepto seminal del enfoque interseccional, da cuenta no sólo 
del hecho de que diferentes tipos de discriminación u opresión acontecen de manera simultánea, sino también del hecho de que el sujeto hegemónico y el sujeto subalterno coexisten en el mismo plano de simultaneidad y no hay lugar, así, para un análisis en términos de precedencia lógica o cronológica. La idea de encrucijada o cruce de caminos alude a la idea de que el sujeto se encuentra siempre en un cruce de varias vías o desigualdades, cada una de ellas apuntando en direcciones políticas e identitarias diferentes; es una idea que pone de relieve la posibilidad de que unas desigualdades puedan ser más importantes para el sujeto que otras y de que los sistemas políticos puedan prestar mayor atención a unas que a otras. Con la noción de encrucijada se moviliza la espinosa cuestión de la visibilidad de las opresiones, ya que apunta claramente al hecho de que en toda sociedad ciertos sistemas de opresión resultan más visibles y comprensibles que otros. La noción de discriminación múltiple trata de neutralizar las concepciones unilaterales que privilegian uno solo de los sistemas de opresión ${ }^{11}$. Las múltiples relaciones entre diferentes categorías de discriminación han dado lugar a una serie de aproximaciones identitarias al sistema de desigualdades, entre las que destaca la noción de "matriz de dominación" de Collins (1990), que se basa en la existencia de una pluralidad de sistemas de opresión entrelazados entre sí. En sentido contrario, Jasbir Puar (2007) aboga por abandonar la aproximación identitaria, que a menudo se limita a crear identidades para a continuación reclamar derechos, y avanzar en su lugar hacia una mejor comprensión de la especificidad personal (corporal, afectiva) del sujeto. La crítica de Puar va dirigida específicamente

11 "A menudo se presenta como una mirada "amable" sobre la inclusión de la diversidad presente en la sociedad [...] por reducirse a evocar diferentes desigualdades obviando cómo las diferentes desigualdades atraviesan a los sujetos y tienen relaciones recíprocas. [...] Es decir, volviendo a concepciones que simplifican la interseccionalidad a una mirada sobre la diversidad de la ciudadanía y las diferentes pujas por obtener reconocimiento, en lugar de entender este concepto como una mera relación entre desigualdades y que evidencia las relaciones de poder" (Platero Méndez 2014: 63). 
contra la utilización en cierto modo perversa que el poder político ha hecho con frecuencia de los enfoques interseccionales de los sistemas de opresión y desigualdad. Por ejemplo, muy a menudo las políticas antidiscriminatorias parecen encaminadas a solucionar los problemas de los grupos hegemónicos y no los de los grupos por los que dicen trabajar. Esto hace que ciertos grupos subyugados o marginales permanezcan en un estado de permanente subordinación, dado que el poder nominalista de la ley que les declara "oprimidos" cae sobre ellos con el peso de las categorías inmutables. Así, se presenta a la persona oprimida en virtud de cierto rasgo identitario como permanentemente en lucha con el sistema ${ }^{12}$.

Un ejemplo que Platero Méndez menciona en su reflexión sobre las posiciones de Jasbir Puar es el de la importancia concedida al matrimonio entre personas del mismo sexo y las consecuencias que este encendido debate tienen al dejar en un segundo término otras necesidades de las personas homosexuales. Así, en el debate sobre el matrimonio homosexual han quedado prácticamente apagadas las voces críticas "que señalan el papel que juegan instituciones como el ejército, la iglesia o las prisiones en la discriminación específica contra las personas LGBTQ", tal como en su tiempo denunciaron colectivos como La Radical Gay y más recientemente hace Against Equality (Platero Méndez 2014: 66). La propia existencia en Madrid de un Orgullo Alternativo desde 2006 resalta la existencia de este tipo de miradas interseccionales que critican la insuficiente atención que la ortodoxia LGBTQ presta a personas que viven bajo múltiples opresiones: migrantes, pobres, discapacitados, etc.

Las dos novelas de Pombo que venimos comentando revelan aspectos interesantes en relación a la interseccionalidad. Como

12 Los conceptos de homonormatividad, homonacionalismo, pink-washing, diáspora queer y otros similares consiguen poner de manifiesto las estrategias que estados y empresas utilizan para presentar una imagen de tolerancia que a veces contradicen con sus políticas (Platero Méndez 2014: 65-66). 
cualquier otra narración gay, ambas novelas describen personajes masculinos que están lejos de detentar la autoridad simbólica comúnmente asociada al heterosexual; lo cual, como comenta Platero Méndez, abre inmediatamente la puerta a una perspectiva interseccional en la que la opresión del homosexual se puede discutir en relación a otras opresiones. Los homosexuales de Pombo, sexófobos y a menudo atormentados, sufren los efectos de otras masculinidades hegemónicas ${ }^{13}$, así como los efectos de un desempoderamiento en todos los órdenes ${ }^{14}$, una negación generalizada de opciones y oportunidades. En Los delitos insignificantes, Ortega desgrana esta acumulación de victimizaciones, algunas de ellas auto-infligidas, conectándolas directamente con una cierta angustia nominalista que recuerda la prehistoria del concepto de homosexualidad, cuando la sodomía era el pecado nefando cuyo nombre no se debía pronunciar; en estas palabras se percibe, también, la angustia de la víctima que se cree culpable de su propia situación:

Nunca me han gustado las mujeres. La única mujer que he conocido, con quien he tenido relaciones [...] me trató muy cruelmente. A mí no me gustan los chicos afeminados. Yo no soy homosexual. ¿Cómo se llama lo que soy? ¡Qué más da cómo se llame!, Ortega se oyó decir a sí mismo. Es que sólo me gusta mirarles. La belleza de los efebos es la única belleza que entiendo. Me gusta mirarles.

13 Las masculinidades pombianas, frecuentemente asociadas a roles subalternos, constituyen un complejo y en cierto modo radical entramado de prácticas susceptibles de ser descritas conforme al paradigma de la masculinidad hegemónica propuesto por Connell y Messerschmidt. La noción de masculinidad hegemónica recurre al concepto gramsciano de hegemonía para describir las prácticas sociales que posibilitan y perpetúan una estructura jerárquica en la que el hombre domina y la mujer es dominada. Si se aplica el mecanismo regulador de la interseccionalidad, el concepto define una estructura jerárquica que se perpetúa no solo por el recurso a la dominación sino también por el consentimiento cultural, la centralidad discursiva, la institucionalización y la marginalización o deslegitimación de alternativas (Connell y Messerschmidt, 2005: 846).

14 Atribuido a un discurso patriarcal que enfatiza la heterosexualidad obligatoria y la primacía de la familia (Perriam et al. 2000: 99). 
Pienso en ellos todo el tiempo. Pero nunca he tenido relaciones con ninguno. Yo soy impotente. Desde el principio, mi vida ha sido un fracaso. Salgo a la calle, no tengo nada que hacer, bebo demasiado. Ayer bebí seis cervezas y tomé diez optalidones. Me paso todo el día en casa, masturbándome. (Pombo 1986:82).

La internalización de la opresión derivada de la homosexualidad, que induce a Ortega a culparse de cuanto le ocurre, lleva también a otros personajes masculinos a establecer entre ellos relaciones punitivas que desbordan, con mucho, el cauce del paradigma sexual. Por ejemplo, Hernández, un amigo escritor tan frustrado como Ortega, con quien éste se cita ocasionalmente para intercambiar lamentos, muestra una homosexualidad tan culpabilizada y vergonzante como la del propio Ortega. En cierta ocasión Ortega trata de ayudarle buscándole un prostituto, pero sólo siente por él compasión y asco. La aparición de personajes masculinos caracterizados como jóvenes, atractivos, de sexualidad ambigua, y dispuestos a prostituirse con los personajes maduros como el Ortega de Los delitos insignificantes o el Salazar de Contra natura abre, naturalmente, otra posibilidad de análisis interseccional. Se trata de relaciones de prostitución que introducen en la dinámica de los relatos las nociones concomitantes de poder, dominación/sumisión y explotación. Sin embargo, la experiencia de Ortega y Salazar al establecer este tipo de relaciones termina por volverse contra ellos: ambos personajes entran en esas relaciones desiguales con cierta curiosidad para caer a continuación en el autoengaño de que existe un amor recíproco y para culminar, en ambos casos, en situaciones de gran violencia que conducen al suicidio del hombre maduro y a la huida del joven.

Si en Los delitos insignificantes se percibe un breve e inexplorado conato de complementación de la figura de Ortega con una imagen en cierto modo especular de sí mismo en la figura de Hernández, en Contra Natura esta posibilidad se desarrolla plenamente mediante la tensión narrativa entre los dos personajes homosexuales principales, Javier Salazar y Paco Allende. Pombo 
se refiere a la pareja Salazar/Allende como la antítesis entre "un hombre contradictorio y cruel y [...] otro que, sin dejar de ser homosexual, alcanza la dignidad del hombre que ama lo que ama" (Pombo en Manrique 2005). El "Epílogo" que pone fin a la novela enfatiza sin ambages la relación de oposición y complementariedad entre ambos, y lo hace no sólo en lo relativo a la narración sino también, principalmente, a lo que podríamos calificar como dimensión ética. Los dos modos radicales de vivir la homosexualidad a los que hace referencia el autor en el "Epílogo" han de ponerse en relación con estos dos personajes.

David Vilaseca argumenta que, si bien la narrativa de Contra natura termina por primar éticamente a Allende por su renuncia al amor físico con Durán, el personaje más claramente radical es el de Javier Salazar, que encarna una respuesta terminal y definitiva al rechazo de Juanjo ${ }^{15}$. El suicidio de Salazar representa el impulso tanático y, con él, la sinthomosexuality o negativismo fundamental que impregna todo lo homosexual (Edelman 2004). No de otra manera ha de entenderse que Vilaseca atribuya a la novela una ética queer radical que va más allá de las diferencias entre personajes específicos: Salazar y Allende alientan un mismo espíritu anti-social y un mismo compromiso con nociones absolutas como la verdad y lo universal que, según puntualiza Vilaseca, sitúan a ambos personajes en la esfera de las causas radicales, lo cual, en un mundo cada vez más materialista y anodino no puede sino ser interpretado como un posicionamiento político y moral (Vilaseca 2010: 259, énfasis en el original).

El clímax narrativo de Contra natura se alcanza nítidamente en el capítulo 49 de los cincuenta de los que consta la novela. La línea argumental centrada en la relación que el acomodado editor jubilado Javier Salazar mantiene con el joven de doble vida

15 Vilaseca subraya la dimensión moral de las palabras que Pombo pone en boca se Salazar, que posiblemente sean las más radicales nunca salidas de la pluma de un miembro de la Real Academia, ya que el personaje defiende la conexión ontológica entre homosexualidad, marginalidad y muerte (Vilaseca 2010: 259-260). 
Juanjo Garnacho alcanza en este capítulo su límite en una escena nocturna y violenta que transcurre en el apartamento de Salazar. La perspectiva con la que arranca el capítulo 49, por su parte, es la de Allende, el amigo que asiste, confuso, a la violenta escena en la que Juanjo Garnacho abusa con impudor de un Salazar anulado por el alcohol; confuso e impotente al comprobar que los acontecimientos siguen una lógica ingobernable, ajena a toda volición, "como si Salazar y Juanjo fueran figuras goyescas, con los ojos vendados, que se atizan mamporros ciegamente en un juego cruel de la gallina ciega" (Pombo 2005a: 534). El perspectivismo narrativo, que ha jugado un papel importante en toda la novela, adquiere gran relevancia en esta escena y parece poner de relieve, una vez más, la pluralidad de las percepciones y de las experiencias; la pluralidad de las identidades que, adoptando un enfoque interseccional, pone de manifiesto la imposibilidad de reducir la violencia a un solo tipo o a una sola fuente. Salazar, que a lo largo de la novela ha hecho gala de actitudes manipuladoras, ejerciendo todo tipo de violencia simbólica sobre su entorno, se encuentra ahora sometido a la dialéctica cruel de la violencia física, un tipo de opresión directa que parece metaforizar todas las violencias indirectas y simbólicas que han ido jalonando la construcción de este personaje ${ }^{16}$.

Los acontecimientos se precipitan: Juanjo agrede a Salazar con gran violencia y le roba sus tarjetas de crédito. Salazar, que creía estar enamorado de Juanjo, recibe la humillación de la paliza y el robo como el episodio que pone fin a su vida:

16 Resulta de gran interés el ejercicio de intertextualidad que Pombo lleva a cabo en Contra natura. Si la construcción de una tradición literaria depende en gran medida del diálogo entre textos, la conciencia homosexual de Pombo parece reclamarse heredera, en esta novela, de la estirpe más clásica de autores occidentales homosexuales. Por eso es muy destacable que en el momento de mayor tensión narrativa de la novela se equipare la muerte de Salazar con la muerte de Passolini, ambas invisibilizadas: "Allende no puede irse ahora porque lo que va a ver nadie lo ha visto nunca. Nadie vio nunca aquel momento en que cayó muerto Passolini en Ostia a manos del chico Pellosi o quizá de dos asesinos a sueldo que contrató la derecha italiana, o quizá la izquierda italiana". (Pombo 2005a: 536). 
Salazar se incorpora un poco [...] Dando tumbos va a la pequeña habitación contigua, donde tiene parte de su biblioteca, que tiene un balcón que se abre a la calle [...] saca medio cuerpo balcón afuera: abajo Juanjo pone en marcha la Yamaha, el Miguel sentado atrás. Salazar pega un grito: ijJuanjo!! Se abalanza al parapeto del balcón con tanta fuerza que el balcón le llega por debajo de la cintura y Salazar, cabeza abajo, quiere desaparecer. Y cae cinco pisos de golpe contra el asfalto dulce de la noche rizomática. (Pombo 2005a: 549-550).

El paralelismo entre el suicidio de Salazar y el suicidio de Ortega en el último párrafo de Los delitos insignificantes no puede ser en modo alguno casual:

Se cerró la puerta de un portazo. La puerta de la calle. No podía pensar nada. Logró pensar por fin: "Esto es lo que buscaba, ¿no es esto? Un final feliz." Salió a la terracilla y miró al sol. Un sol todavía fuerte. Todavía con tiempo por delante. Las losetas de baldosín rojo estaban cálidas como la piel de un animal apacible. Se encaramó a caballo sobre la barandilla, como de niño. Se abalanzó al vacío, ladeado, como un saco de noventa kilos de carne. Ilegible es el sol desvinculador del mundo. (Pombo 1986: 234).

Esa última frase ("Ilegible es el sol desvinculador del mundo") había constituido, en sí misma, uno de los poemas incluidos en Hacia una constitución poética del año en curso (Pombo 1980, poema 2.8). Años después Pombo habría de reflexionar ${ }^{17}$ sobre el origen de ese poema que en cierto modo delata una influencia de Eliot y su espanto ante la incoherencia del mundo, llegando incluso a citar de memoria una imagen de The Waste Land: "On Margate Sands, I I can connect / Nothing with nothing / The broken fingernails of dirty hands / My people humble people who expect / Nothing"18. Hay un innegable aire de familia entre esa imagen ("I

17 Pombo en "Conversaciones nada académicas: Álvaro Pombo y José Antonio Marina, sobremesa en Las Jaras", El Cultural, 2/1/2003.

18 T.S. Eliot, “The Fire Sermon: On Margate Sands”, Part III of The Waste Land (1922). 
can connect / Nothing with nothing") y la ilegibilidad desvinculadora, nihilista, de Pombo, que de modo novelescamente sorprendente, inesperado, subsume en sí misma todo el planteamiento estético, narrativo y moral de la novela. Pero la incoherencia e ilegibilidad del mundo de Los delitos insignificantes se trasforma en una "noche rizomática" en Contra natura: la imagen de la incoherencia se ve reemplazada por la imagen de la conectividad fundamental, el rizoma. La visión totalizadoramente nihilista deja paso a una de las imágenes que con más acierto captura las complejidades de la interseccionalidad.

La repetición del desenlace trágico en novelas separadas por casi dos décadas es un indicio suficientemente esclarecedor de lo que Pombo entiende por la complejidad de la experiencia homosexual. Frente al banal hedonismo que a veces destila el consumismo gay, el destino del homosexual pasa, para Pombo, por una experiencia directa de la marginación y la otredad. Resuenan en estas líneas de Pombo las controvertidas tesis de Lee Edelman sobre la necesidad de preservar lo queer como una ética contraria a la normalización, como una alternativa al imperativo patriarcal de construir el futuro a partir de la creación y adoctrinamiento de las generaciones futuras, empezando por la generación inmediatamente posterior que literariamente suele personificarse en la figura del niño. La alternativa que lo queer representa viene a ser, para Edelman, un negativo de la cultura heteronormativa hegemónica, y de hecho atribuye a la homosexualidad una carga de negatividad que, desde su punto de vista, no solamente no debería ser rechazada sino que debería constituir la columna vertebral de un activismo queer con un claro e irrenunciable compromiso anti-normalizador (Edelman 2004). En Contra natura, Javier Salazar es un personaje-tesis que encarna y verbaliza esta filosofía radicalmente tanática de la homosexualidad en la que palabra "muerte" aparece hasta tres veces: 
En mi conciencia, como en la de Jean Genet o en la de Sartre, la homosexualidad, su teoría y sobre todo su práctica, conecta ontológicamente con la marginación y con la soledad y con la muerte y con las cárceles. Ontológicamente significa $a b$ ovo: significa antes y después de toda aceptación jurídica o política o social. Nadie nos librará jamás de nuestra esencial conexión con la marginación, con el fracaso y con la muerte. La mayor parte de la gracia que aún tenemos los maricas, antes que la trivialidad y la normalidad nos conviertan en simples consumidores pancistas españoles, mariquitas per cápita que contribuyen con normalidad e incluso con un muy buen balance anual a los gastos de la hacienda pública, antes y después de toda esa babosa voluntad de normalización e identidad con los comemierdas que siempre hemos envidiado y odiado, nuestra conexión más pura es con el fracaso, con la marginación y con la muerte. (Pombo 2005a: 531-532) ${ }^{19}$.

A modo de conclusión, necesariamente provisional, proponemos avanzar la hipótesis de que la pluralidad de perspectivas que Pombo adopta para su construcción narrativa de la experiencia homosexual se ve notablemente enriquecida a través de una lectura interseccional. La aparente paradoja de aplicar un concepto procedente del feminismo afroamericano al estudio de homosexualidades españolas no lo es tanto si se tiene en cuenta el radicalismo ético del que se nutre el enfoque interseccional y que está en la base misma de la poética pombiana. Aunque en España abunda un tipo de literatura de consumo gay que poco o nada tiene que ver con los planteamientos interseccionales, la de Pombo se sitúa en su mismo epicentro al vindicar la proliferación rizomática como el modelo para pensar la homosexualidad en tanto que pluralidad e irreductible a cualquier noción de norma o normalización. Hemos señalado varios ejemplos de encrucijadas y múltiples opresiones homosexuales en Los delitos

19 Y el propio Pombo añade, en el "Epílogo": "Frente a los años de lucha por los derechos gays hemos llegado a un tiempo - admirable en muchos sentidosen que lo gay comienza a trivializarse. Hay un proceso trivializador que afecta a nuestras juventudes y que se confunde con el consumismo y con el hedonismo de nuestra sociedad española actual." (Pombo 2005a: 560). 
insignificantes y Contra natura: la oposición joven/viejo, siempre resuelta en términos de una incompatibilidad en la que la edad se convierte en un motivo más de discriminación; la oposición dominante/sumiso; las relaciones punitivas entre homosexuales, explícita o tácitamente explicadas como consecuencia de la homofobia internalizada; los desdoblamientos especulares de personajes que se presentan como opuestos y complementarios; y la asunción de la homosexualidad como negativismo fundamental relacionado con la pulsión de muerte. Contra natura fue ampliamente comentada en su momento como un alegato contra la superficialidad y como una defensa de la autenticidad de la experiencia homosexual. Tras el somero análisis del peso que el enfoque interseccional tiene en esta valoración resulta innegable que la ética anti-normalizadora de Pombo está en la base de su interés por la exploración de la experiencia homosexual como garante de la posibilidad de liberación profunda, personal, y por lo tanto infinitamente variada. El recurso literario a personajes tipificados y previsibles, que Pombo utilizó en los comienzos de su carrera y que todavía se percibe en Los delitos insignificantes, deja paso a una galería más amplia de personajes complejos, multidimensionales, en Contra natura. La coexistencia de múltiples ejes identitarios en los cuatro personajes principales de esta novela responde con precisión a las ideas interseccionales de encrucijada y simultaneidad, y nos pone por lo tanto sobre la pista de lo que podríamos definir como una poética pombiana de la opresión. Desde este punto de vista, Contra natura representa una extraordinaria vuelta de tuerca a las categorías que habitualmente se han utilizado para clasificar la literatura de Pombo (los ciclos de la falta de sustancia y de la realidad, los ciclos negativo y positivo de los que habla José Antonio Marina) ya que el sistema de múltiples opresiones que define a cada personaje se transforma en una lente que hace visibles conciencias y experiencias de otro modo inefables. Sin olvidar, con Puar, que esta estrategia no está libre del riesgo de contribuir a la perpetuación de las desigualdades, lo cierto es que el detalle con el que Pombo disecciona la 
homosexualidad masculina de la España contemporánea eleva a Contra natura a un nivel político, ético y literario muy diferente al alcanzado por Los delitos insignificantes y por la mayor parte de la literatura homosexual española.

\section{Referencias bibliográficas}

Aliaga, Juan Vicente (1997), "Víctimas y depravados: una paranoia heterosexual", en Aliaga, Juan Vicente y José Miguel G. Cortés, Identidad y diferencia: sobre la cultura gay en España, Barcelona, Madrid, Editorial Gay y Lesbiana, pp. 74-88.

Collins, Patricia H. (1990), Black Feminist Thought: Knowledge, Consciousness and the Politics of Empowerment, Boston, Unwin Hyman.

Connell, R. W. y James W. Messerschmidt (2005), “Hegemonic Masculinity: Rethinking the Concept», Gender and Society, 19, 6, pp. 829-859.

Edelman, Lee (2004) No Future: Queer Theory and the Death Drive, Durham, Duke University Press.

Eymar, Marcos (2006), "Contra natura, de Álvaro Pombo", El Ciervo, 660, p. 42.

García Sánchez, J. (1979), “Los protocolos de Álvaro Pombo", El Viejo Topo, 34.

Lamo de Espinosa, E. (1989), Delitos sin víctima, Madrid, Alianza Universidad.

Manrique, Winston (2005), "La condena de la Iglesia a la homosexualidad es frívola: entrevista a Álvaro Pombo", El País, $1 / 12 / 2005$.

Marina, José Antonio (2006), "Pombo", Boletín de la Fundación Juan March, 354, p. 10.

Martínez Expósito, Alfredo (2004), Escrituras Torcidas: ensayos de crítica "queer", Barcelona, Laertes. 
Martínez Expósito, Alfredo (2013), “Queer Literature in Spain: Pathways to Normalisation", Culture and History Digital Journal, 2/1, e010, 011-019.

Mira, Alberto (1999), Para entendernos: diccionario de cultura homosexual, gay y lésbica, Barcelona, Ediciones de la Tempestad.

Morán, David (2005) "Álvaro Pombo afirma que Contra natura es una novela ejemplar", $A B C, 20 / 12 / 2005$.

Pérez SÁnchez, Gema (2014) "Spanish Literature in the Long Twentieth Century, 1898-2007", McCallum, E. L y Mikko Tuhkanen, eds., The Cambridge History of Gay and Lesbian Literature, Cambridge University Press, pp. 438-458.

Perriam, Chris, Michael Thompson, Susan Frenk y Vanessa Knights (2000) A New History of Spanish Writing 1939 to the 1990s, Oxford University Press.

Platero Méndez, Raquel (Lucas) (2014) “Metáforas y articulaciones para una pedagogía crítica sobre la interseccionalidad", Quaderns de Psicologia, 16/1, pp. 55-72.

Pомвo, Álvaro (1973) Protocolos. Madrid, Biblioteca Nueva.

Ромво, Álvaro (1977) Relatos sobre la falta de sustancia, Barcelona, Anagrama. Existe edición crítica de Mario Crespo López (Madrid, Cátedra, 2013).

Pombo, Álvaro (1980) Hacia una constitución poética del año en curso, Barcelona, La Gaya Ciencia.

Ромвo, Álvaro (1985) “De las narraciones y sus filosofías furtivas", Revista de Occidente, 44, pp. 7-17.

Ромво, Álvaro (1986) Los delitos insignificantes, Barcelona, Anagrama.

Ромвo, Álvaro (1990) El metro de platino iridiado, Barcelona, Anagrama. 
Pombo, Álvaro (1996) Vida de san Francisco de Asís: una paráfrasis, Barcelona, Planeta.

Pombo, Álvaro (1999) La cuadratura del círculo, Barcelona, Anagrama.

Pombo, Álvaro (2001) El cielo raso, Barcelona, Anagrama.

Ромво, Álvaro (2004), Verosimilitud y verdad, Real Academia Española, Madrid.

Pombo, Álvaro (2005a), Contra Natura, Barcelona, Anagrama.

Ромвo, Álvaro (2005b), “Llamar al matrimonio por su nombre", Archipiélago: Cuadernos de crítica de la cultura, 67, pp. 107-113.

Pozuelo Yvancos, José María (2006), “¿Quién teme a la literatura homosexual?", $A B C, 8 / 1 / 2006$.

Prádanos, Luis I. (2006), "Álvaro Pombo: Contra natura”, Hispania, 89/4, pp. 908-909.

PuAr, Jasbir K. (2007), Terrorist Assemblages: Homonationalism in Queer Times, Durham, Duke University Press.

Rocamora, Jesús (2009), "Yo no soy gay: soy pre-gay”, Público, 15/8/2009.

Undurraga, Vicente (2011), "Soy un homosexual homófobo", The Clinic, 31/3/2011.

Vilaseca, David (2010), "Queer Ethics and the Event of Love in Álvaro Pombo's Contra natura (2005)", Revista Canadiense de Estudios Hispánicos, 35/1, pp. 243-263.

Villena, Luis Antonio de (2006), “Álvaro Pombo, feminólogo", El País, 11/11/2006.

Alfredo Martínez Expósito

University of Melbourne 